\title{
Some New Results on Prime Cordial Labeling
}

\author{
S. K. Vaidya ${ }^{1}$ and N. H. Shah ${ }^{2}$ \\ ${ }^{1}$ Saurashtra University, Rajkot, Gujarat 360005, India \\ ${ }^{2}$ Government Polytechnic, Rajkot, Gujarat 360003, India \\ Correspondence should be addressed to S. K. Vaidya; samirkvaidya@yahoo.co.in
}

Received 7 January 2014; Accepted 18 February 2014; Published 23 March 2014

Academic Editors: E. Manstavicius, J. Rada, and F. Rosselló

Copyright (C) 2014 S. K. Vaidya and N. H. Shah. This is an open access article distributed under the Creative Commons Attribution License, which permits unrestricted use, distribution, and reproduction in any medium, provided the original work is properly cited.

A prime cordial labeling of a graph $G$ with the vertex set $V(G)$ is a bijection $f: V(G) \rightarrow\{1,2,3, \ldots,|V(G)|\}$ such that each edge $u v$ is assigned the label 1 if $\operatorname{gcd}(f(u), f(v))=1$ and 0 if $\operatorname{gcd}(f(u), f(v))>1$; then the number of edges labeled with 0 and the number of edges labeled with 1 differ by at most 1 . A graph which admits a prime cordial labeling is called a prime cordial graph. In this work we give a method to construct larger prime cordial graph using a given prime cordial graph $G$. In addition to this we have investigated the prime cordial labeling for double fan and degree splitting graphs of path as well as bistar. Moreover we prove that the graph obtained by duplication of an edge (spoke as well as rim) in wheel $W_{n}$ admits prime cordial labeling.

\section{Introduction}

We consider a finite, connected, undirected, and simple graph $G=(V(G), E(G))$ with $p$ vertices and $q$ edges which is also denoted as $G(p, q)$. For standard terminology and notations related to graph theory we follow Balakrishnan and Ranganathan [1] while for any concept related to number theory we refer to Burton [2]. In this section we provide brief summary of definitions and other required information for our investigations.

Definition 1. The Graph labeling is an assignment of numbers to the vertices or edges or both subject to certain condition(s). If the domain of the mapping is the set of vertices (edges), then the labeling is called a vertex labeling (edge labeling).

Many labeling schemes have been introduced so far and they are explored as well by many researchers. Graph labelings have enormous applications within mathematics as well as to several areas of computer science and communication networks. Various applications of graph labeling are reported in the work of Yegnanaryanan and Vaidhyanathan [3]. For a dynamic survey on various graph labeling problems along with an extensive bibliography we refer to Gallian [4].
Definition 2. A labeling $f: V(G) \rightarrow\{0,1\}$ is called binary vertex labeling of $G$ and $f(v)$ is called the label of the vertex $v$ of $G$ under $f$.

Notation 1. If for an edge $e=u v$, the induced edge labeling $f^{*}: E(G) \rightarrow\{0,1\}$ is given by $f^{*}(e)=|f(u)-f(v)|$. Then

$$
\begin{array}{r}
v_{f}(i)=\text { number of vertices of } G \text { having label } i \text { under } f \\
e_{f}(i)=\text { number of edges of } G \text { having label } i \text { under } f^{*}, \\
\text { where } i=0 \text { or } 1 .
\end{array}
$$

Definition 3. A binary vertex labeling $f$ of a graph $G$ is called a cordial labeling if $\left|v_{f}(0)-v_{f}(1)\right| \leq 1$ and $\left|e_{f}(0)-e_{f}(1)\right| \leq 1$. A graph $G$ is cordial if it admits cordial labeling.

The concept of cordial labeling was introduced by Cahit [5].

The notion of prime labeling was originated by Entringer and was introduced by Tout et al. [6].

Definition 4. A prime labeling of a graph $G$ is an injective function $f: V(G) \rightarrow\{1,2, \ldots,|V(G)|\}$ such that for, every 
pair of adjacent vertices $u$ and $v, \operatorname{gcd}(f(u), f(v))=1$. The graph which admits a prime labeling is called a prime graph.

The concept of prime labeling has attracted many researchers as the study of prime numbers is of great importance because prime numbers are scattered and there are arbitrarily large gaps in the sequence of prime numbers. Vaidya and Prajapati [7, 8] have investigated many results on prime labeling. Same authors [9] have discussed prime labeling in the context of duplication of graph elements. Motivated through the concepts of prime labeling and cordial labeling, a new concept termed as a prime cordial labeling was introduced by Sundaram et al. [10] which contains blend of both the labelings.

Definition 5. A prime cordial labeling of a graph $G$ with vertex set $V(G)$ is a bijection $f: V(G) \rightarrow\{1,2,3, \ldots,|V(G)|\}$ and if the induced function $f^{*}: E(G) \rightarrow\{0,1\}$ is defined by

$$
\begin{aligned}
f^{*}(e=u v) & =1, \quad \text { if } \operatorname{gcd}(f(u), f(v))=1, \\
& =0, \quad \text { otherwise }
\end{aligned}
$$

then the number of edges labeled with 0 and the number of edges labeled with 1 differ by at most 1 . A graph which admits prime cordial labeling is called a prime cordial graph.

Many graphs are proved to be prime cordial in the work of Sundaram et al. [10]. Prime cordial labeling for some cycle related graphs has been discussed by Vaidya and Vihol [11]. Prime cordial labeling in the context of some graph operations has been discussed by Vaidya and Vihol [12] and Vaidya and Shah $[13,14]$. Vaidya and Shah [14] have proved that the wheel graph $W_{n}$ admits prime cordial labeling for $n \geq 8$ while same authors in [15] have discussed prime cordial labeling for some wheel related graphs. Babitha and Baskar Babujee [16] have exhibited prime cordial labeling for some cycle related graphs and discussed the duality of prime cordial labeling. The same authors in [17] have derived some characterizations of prime cordial graphs and investigated various methods to construct larger prime cordial graphs using existing prime cordial graphs. We investigate a method different from existing one to construct larger prime cordial graph from an existing prime cordial graph.

Definition 6. The wheel $W_{n}$ is defined to be the join $K_{1}+C_{n}$. The vertex corresponding to $K_{1}$ is known as apex and vertices corresponding to cycle are known as rim vertices while the edges corresponding to cycle are known as rim edges.

Definition 7. The bistar $B_{n, n}$ is a graph obtained by joining the center (apex) vertices of two copies of $K_{1, n}$ by an edge.

Definition 8. The fan $F_{n}$ is the graph obtained by taking $n-2$ concurrent chords in cycle $C_{n+1}$. The vertex at which all the chords are concurrent is called the apex vertex. In other words, $F_{n}=P_{n}+K_{1}$.

Definition 9. The double fan $\mathrm{DF}_{n}$ consists of two fan graphs that have a common path. In other words, $\mathrm{DF}_{n}=P_{n}+\bar{K}_{2}$.
Definition 10. The duplication of an edge $e=u v$ of graph $G$ produces a new graph $G^{\prime}$ by adding an edge $e^{\prime}=u^{\prime} v^{\prime}$ such that $N\left(u^{\prime}\right)=N(u) \cup\left\{v^{\prime}\right\}-\{v\}$ and $N\left(v^{\prime}\right)=N(v) \cup\left\{u^{\prime}\right\}-\{u\}$.

Definition 11 (see [18]). Let $G=(V(G), E(G))$ be a graph with $V=S_{1} \cup S_{2} \cup S_{3} \cup \cdots S_{t} \cup T$, where each $S_{i}$ is a set of vertices having at least two vertices of the same degree and $T=$ $V \backslash \cup_{i=1}^{t} S_{i}$. The degree splitting graph of $\mathrm{G}$ denoted by $D S(G)$ is obtained from $G$ by adding vertices $w_{1}, w_{2}, w_{3}, \ldots, w_{t}$ and joining to each vertex of $S_{i}$ for $1 \leq i \leq t$.

\section{Main Results}

Theorem 12. Let $G(p, q)$ with $p \geq 4$ be a prime cordial graph and let $K_{2, n}$ be a bipartite graph with bipartition $V=V_{1} \cup V_{2}$ with $V_{1}=\left\{v_{1}, v_{2}\right\}$ and $V_{2}=\left\{u_{1}, u_{2}, \ldots, u_{n}\right\}$. If $G_{1}$ is the graph obtained by identifying the vertices $v_{1}$ and $v_{2}$ of $K_{2, n}$ with the vertices of $G$ having labels 2 and 4 , respectively, then $G_{1}$ admits prime cordial labeling in any of the following cases:

(i) $n$ is even and $G$ is of any size q;

(ii) $n, p$, and $q$ are odd with $e_{f}(0)=\lfloor q / 2\rfloor$;

(iii) $n$ is odd, $p$ is even, and $q$ is odd with $e_{f}(0)=\lceil q / 2\rceil$.

Proof. Let $G(p, q)$ be a prime cordial graph and let $f_{1}$ be the prime cordial labeling of $G$. Let $w_{1}, w_{2} \in V$ be the vertices of $G$ such that $f_{1}\left(w_{1}\right)=2$ and $f_{1}\left(w_{2}\right)=4$. Consider the $K_{2, n}$ with bipartition $V=V_{1} \cup V_{2}$ with $V_{1}=\left\{v_{1}, v_{2}\right\}$ and $V_{2}=$ $\left\{u_{1}, u_{2}, \ldots, u_{n}\right\}$. Now identify the vertices $v_{1}$ to $w_{1}$ and $v_{2}$ to $w_{2}$ and denote the resultant graph as $G_{1}$. Then $V\left(G_{1}\right)=V(G) \cup$ $\left\{u_{1}, u_{2}, \ldots, u_{n}\right\}$ and $E\left(G_{1}\right)=E(G) \cup\left\{w_{1} u_{i}, w_{2} u_{i} / 1 \leq i \leq n\right\}$ so $\left|V\left(G_{1}\right)\right|=p+n$ and $\left|E\left(G_{1}\right)\right|=q+2 n$. To define $f: V\left(G_{1}\right) \rightarrow$ $\{1,2,3, \ldots, p+n\}$, we consider the following three cases.

Case (i) ( $n$ is even and $G$ is of any size $q$ ). Since $G$ is a prime cordial graph, we assign vertex labels such that $f\left(w_{i}\right)=$ $f_{1}\left(w_{i}\right)$, where $w_{i} \in V(G)=V\left(G_{1}\right) \cap V(G)$ and $i \leq i \leq p$ :

$$
f\left(u_{i}\right)=p+i, \quad i \leq i \leq n .
$$

Since $n$ is even and $f_{1}\left(w_{1}\right)=2$ and $f_{1}\left(w_{2}\right)=4, w_{1}$ and $w_{2}$ are adjacent to each $u_{i}, i \leq i \leq n$. And this vertex assignment generates $n$ edges with label 1 and $n$ edges with label 0 . Following Table 1 gives edge condition for prime cordial labeling for $G_{1}$ under $f$.

From Table 1, we have $\left|e_{f}(0)-e_{f}(1)\right| \leq 1$.

Case (ii) ( $n, p$, and $q$ are odd with $\left.e_{f}(0)=\lfloor q / 2\rfloor\right)$. Here $p$ and $q$ both are odd and $G$ is a prime cordial graph with $e_{f}(0)=$ $\lfloor q / 2\rfloor$.

Since $G$ is a prime cordial graph, we keep the vertex label of all the vertices of $G$ in $G_{1}$ as it is. Therefore $f\left(w_{i}\right)=f_{1}\left(w_{i}\right)$, where $w_{i} \in V(G)$ and $i \leq i \leq p$ :

$$
f\left(u_{i}\right)=p+i, \quad i \leq i \leq n .
$$

Since $n$ is odd and $f_{1}\left(w_{1}\right)=2$ and $f_{1}\left(w_{2}\right)=4, w_{1}$ and $w_{2}$ are adjacent to each $u_{i}, i \leq i \leq n$. And this vertex assignment generates $n+1$ edges with label 0 and $n-1$ edges with label 1 . 
TABLE 1

\begin{tabular}{ccc}
\hline$q$ & Edge conditions for $G$ & Edge conditions for $G_{1}$ \\
\hline Even & $e_{f}(0)=e_{f}(1)=\frac{q}{2}$ & $e_{f}(0)=e_{f}(1)=\frac{q}{2}$ \\
& $e_{f}(0)-1=e_{f}(1)=\left\lfloor\frac{q}{2}\right\rfloor$ & $e_{f}(0)-1=e_{f}(1)=\left\lfloor\frac{q}{2}\right\rfloor+n$ \\
Odd & & \\
& $e_{f}(0)=e_{f}(1)-1=\left\lfloor\frac{q}{2}\right\rfloor$ & $e_{f}(0)=e_{f}(1)-1=\left\lfloor\frac{q}{2}\right\rfloor+n$ \\
\hline
\end{tabular}

Therefore edge conditions for $G_{1}$ under $f$ are $e_{f}(0)=$ $\lfloor q / 2\rfloor+n+1$ and $e_{f}(1)=\lceil q / 2\rceil+n-1$. Therefore, $e_{f}(0)-1=$ $e_{f}(1)$. Hence, $\left|e_{f}(0)-e_{f}(1)\right| \leq 1$ for graph $G_{1}$.

Case (iii) ( $n$ is odd, $p$ is even, and $q$ is odd with $e_{f}(0)=\lceil q / 2\rceil$ ). Here $p$ is even, $q$ is odd, and $G$ is a prime cordial graph with $e_{f}(0)=\lceil q / 2\rceil$.

Since $G$ is a prime cordial graph, we keep the vertex label of all the vertices of $G$ in $G_{1}$ as it is. Therefore $f\left(w_{i}\right)=f_{1}\left(w_{i}\right)$, where $w_{i} \in V(G)$ and $i \leq i \leq p$ :

$$
f\left(u_{i}\right)=p+i, \quad i \leq i \leq n .
$$

Since $n$ is odd and $f_{1}\left(w_{1}\right)=2$ and $f_{1}\left(w_{2}\right)=4, w_{1}$ and $w_{2}$ are adjacent to each $u_{i}, i \leq i \leq n$. And this vertex assignment generates $n-1$ edges with label 0 and $n+1$ edges with label 1 .

Therefore edge conditions for $G_{1}$ under $f$ are $e_{f}(0)=$ $\lceil q / 2\rceil+n-1$ and $e_{f}(1)=\lfloor q / 2\rfloor+n+1$. Therefore, $e_{f}(0)=$ $e_{f}(1)-1$. Hence, $\left|e_{f}(0)-e_{f}(1)\right| \leq 1$ for graph $G_{1}$.

Hence, in all the cases discussed above, $G_{1}$ admits prime cordial labeling.

Illustration 1. Consider the graph $G$ as shown in Figure 1, with $p=7$ and $q=9$. $G$ is a prime cordial graph with $e_{f}(0)=4, e_{f}(1)=5$. Take $n=3$ and construct graph $G_{1}$. In accordance with Case (ii) of Theorem 12, a prime cordial labeling of $G_{1}$ is as shown in Figure 1 . Here $e_{f}(0)=8, e_{f}(1)=$ 7.

Theorem 13. Double fan $D F_{n}$ is a prime cordial graph for $n=8$ and $n \geq 10$.

Proof. Let $\mathrm{DF}_{n}$ be the double fan with apex vertices $u_{1}, u_{2}$ and $v_{1}, v_{2}, \ldots, v_{n}$ are vertices common path. Then $\left|V\left(\mathrm{DF}_{n}\right)\right|=n+$ 2 and $\left|E\left(\mathrm{DF}_{n}\right)\right|=3 n-1$. To define $f: V(G) \rightarrow\{1,2,3, \ldots, n+$ $2\}$, we consider the following five cases.

Case 1 ( $n=3$ to 7 and $n=9$ ). In order to satisfy the edge condition for prime cordial labeling in $\mathrm{DF}_{3}$ it is essential to label four edges with label 0 and four edges with label 1 out of eight edges. But all the possible assignments of vertex labels will give rise to 0 labels for at most one edge and 1 label for at least seven edges. That is, $\left|e_{f}(0)-e_{f}(1)\right|=6>1$. Hence, $\mathrm{DF}_{3}$ is not a prime cordial graph.

In order to satisfy the edge condition for prime cordial labeling in $\mathrm{DF}_{4}$ it is essential to label five edges with label 0 and six edges with label 1 out of eleven edges. But all the possible assignments of vertex labels will give rise to 0 labels for at most three edges and 1 label for at least eight edges. That is, $\left|e_{f}(0)-e_{f}(1)\right|=5>1$. Hence, $\mathrm{DF}_{4}$ is not a prime cordial graph.

In order to satisfy the edge condition for prime cordial labeling in $\mathrm{DF}_{5}$ it is essential to label seven edges with label 0 and seven edges with label 1 out of fourteen edges. But all the possible assignments of vertex labels will give rise to 0 labels for at most four edges and 1 label for at least ten edges. That is, $\left|e_{f}(0)-e_{f}(1)\right|=6>1$. Hence, $\mathrm{DF}_{5}$ is not a prime cordial graph.

In order to satisfy the edge condition for prime cordial labeling in $\mathrm{DF}_{6}$ it is essential to label eight edges with label 0 and nine edges with label 1 out of seventeen edges. But all the possible assignments of vertex labels will give rise to 0 labels for at most six edges and 1 label for at least eleven edges. That is, $\left|e_{f}(0)-e_{f}(1)\right|=5>1$. Hence, $\mathrm{DF}_{6}$ is not a prime cordial graph.

In order to satisfy the edge condition for prime cordial labeling in $\mathrm{DF}_{7}$ it is essential to label ten edges with label 0 and ten edges with label 1 out of twenty edges. But all the possible assignments of vertex labels will give rise to 0 labels for at most eight edges and 1 label for at least twelve edges. That is, $\left|e_{f}(0)-e_{f}(1)\right|=4>1$. Hence, $\mathrm{DF}_{7}$ is not a prime cordial graph.

In order to satisfy the edge condition for prime cordial labeling in $\mathrm{DF}_{9}$ it is essential to label thirteen edges with label 0 and thirteen edges with label 1 out of twenty-six edges. But all the possible assignments of vertex labels will give rise to 0 labels for at most twelve edges and 1 label for at least fourteen edges. That is, $\left|e_{f}(0)-e_{f}(1)\right|=2>1$. Hence, $\mathrm{DF}_{9}$ is not a prime cordial graph.

Case $2(n=8,10,11,12)$. For $n=8, f\left(u_{1}\right)=2, f\left(u_{2}\right)=6$ and $f\left(v_{1}\right)=4, f\left(v_{2}\right)=8, f\left(v_{3}\right)=3, f\left(v_{4}\right)=9, f\left(v_{5}\right)=10$, $f\left(v_{6}\right)=5, f\left(v_{7}\right)=7$, and $f\left(v_{8}\right)=1$. Then $e_{f}(0)=11, e_{f}(1)=$ 12.

For $n=10, f\left(u_{1}\right)=2, f\left(u_{2}\right)=6$ and $f\left(v_{1}\right)=3, f\left(v_{2}\right)=$ 9, $f\left(v_{3}\right)=12, f\left(v_{4}\right)=8, f\left(v_{5}\right)=4, f\left(v_{6}\right)=10, f\left(v_{7}\right)=1$, $f\left(v_{8}\right)=5, f\left(v_{9}\right)=7$, and $f\left(v_{10}\right)=11$. Then $e_{f}(0)=15$, $e_{f}(1)=14$

For $n=11, f\left(u_{1}\right)=2, f\left(u_{2}\right)=6$ and $f\left(v_{1}\right)=3, f\left(v_{2}\right)=$ 9, $f\left(v_{3}\right)=12, f\left(v_{4}\right)=8, f\left(v_{5}\right)=4, f\left(v_{6}\right)=10, f\left(v_{7}\right)=5$, $f\left(v_{8}\right)=7, f\left(v_{9}\right)=11, f\left(v_{10}\right)=13$, and $f\left(v_{11}\right)=1$. Then $e_{f}(0)=16, e_{f}(1)=16$.

For $n=12, f\left(u_{1}\right)=2, f\left(u_{2}\right)=6$ and $f\left(v_{1}\right)=3, f\left(v_{2}\right)=$ 9, $f\left(v_{3}\right)=12, f\left(v_{4}\right)=8, f\left(v_{5}\right)=4, f\left(v_{6}\right)=14, f\left(v_{7}\right)=10$, $f\left(v_{8}\right)=5, f\left(v_{9}\right)=7, f\left(v_{10}\right)=11, f\left(v_{11}\right)=13$, and $f\left(v_{12}\right)=$ 1 . Then $e_{f}(0)=18, e_{f}(1)=17$.

Now for the remaining three cases let

$$
\begin{array}{rlrl}
k & =\left\lfloor\frac{n+2}{2}\right\rfloor, & m=\left\lfloor\frac{n+2}{3}\right\rfloor, \\
t_{1}=\left\lceil\frac{3 n-1}{2}\right\rceil, & t_{2}=3 k-7+\left\lceil\frac{m}{2}\right\rceil,
\end{array}
$$

$t_{3}=$ largest even number $\leq n+2$, and $t_{4}=$ largest odd number $\leq n+2$.

Case $3\left(t_{1}=t_{2}\right)$. Consider

$$
f\left(u_{1}\right)=2, \quad f\left(u_{2}\right)=6 .
$$




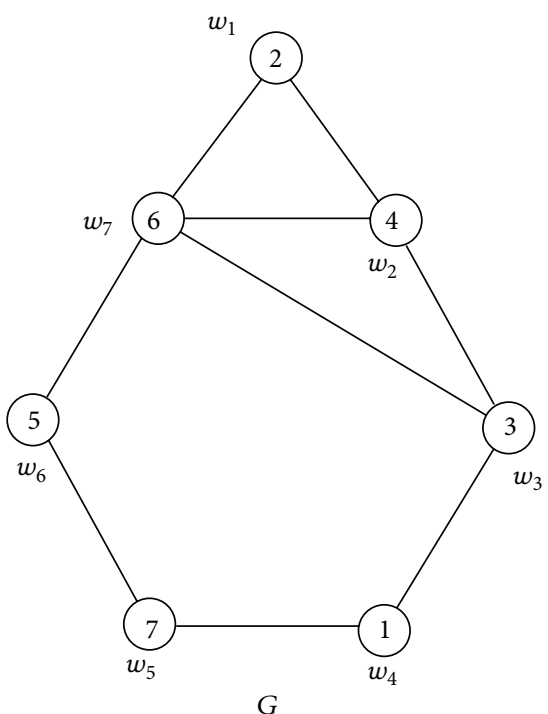

(a)

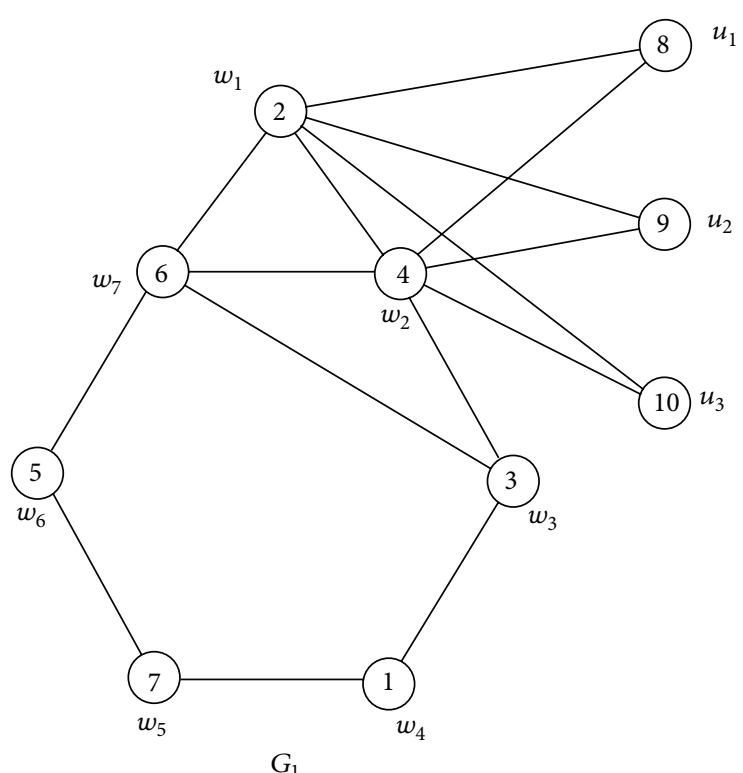

(b)

Figure 1

For the vertices $v_{1}, v_{2}, v_{3}, \ldots, v_{n}$ we assign the vertex labels in the following order: $1, t_{3}, t_{3}-2, t_{3}-4, \ldots, 14,12,10,8,4,3$ $, 5,7,9, \ldots, t_{4}-2, t_{4}$.

Case $4\left(t_{1}>t_{2}\right)$. Consider

$$
f\left(u_{1}\right)=2, \quad f\left(u_{2}\right)=6 .
$$

Let $t_{5}=t_{1}-t_{2}$. Consider

$$
\begin{aligned}
f\left(v_{1}\right) & =3, \\
f\left(v_{2}\right) & =9, \\
f\left(v_{3}\right) & =15, \\
f\left(v_{4}\right) & =21, \\
& \vdots \\
f\left(v_{t_{5}}\right) & =3\left(2 t_{5}-1\right), \\
f\left(v_{t_{5}+1}\right) & =f\left(v_{t_{5}}\right)+6 .
\end{aligned}
$$

Now for remaining vertices $v_{t_{5}+2}, v_{t_{5}+3}, \ldots, v_{n}$ assign the labels $1, t_{3}, t_{3}-2, t_{3}-4, \ldots, 14,12,10,8,4,5,7, \ldots$, all the odd numbers in ascending order.

Case $5\left(t_{2}>t_{1}\right)$. Let $t_{6}=t_{2}-t_{1}$.

Sub-Case 1. $n$ is even. Consider

$$
f\left(u_{1}\right)=2, \quad f\left(u_{2}\right)=6 .
$$

For the vertices $v_{1}, v_{2}, v_{3}, \ldots, v_{n}$ we assign the vertex labels in the following order: $n+2, n+1, n, n-1, n-2, n-3, n+2-2 t_{6}$, $n+2-2\left(t_{6}+1\right), n+2-2\left(t_{6}+2\right), \ldots, 10,8,4,3,5,7, \ldots$ remaining odd numbers in ascending order.

Sub-Case 2. $n$ is odd. Consider

$$
f\left(u_{1}\right)=2, \quad f\left(u_{2}\right)=6 .
$$

For the vertices $v_{1}, v_{2}, v_{3}, \ldots, v_{n}$ we assign the vertex labels in the following order: $n+2, n+1, n, n-1, n-2, n-3, n+2-2 t_{6}$, $n+2-2\left(t_{6}+1\right), n+2-2\left(t_{6}+2\right), \ldots, 10,8,4,3,5,7, \ldots$ remaining odd numbers in ascending order.

In view of the above defined labeling pattern for Cases 3 , 4 , and 5 , we have

$$
e_{f}(0)=\left\lceil\frac{3 n-1}{2}\right\rceil, \quad e_{f}(1)=\left\lfloor\frac{3 n-1}{2}\right\rfloor .
$$

Thus, we have $\left|e_{f}(0)-e_{f}(1)\right| \leq 1$. 10.

Hence, $\mathrm{DF}_{n}$ is a prime cordial graph for $n=8$ and $n \geq$

Illustration 2. For the graph $\mathrm{DF}_{15},\left|V\left(\mathrm{DF}_{15}\right)\right|=17$ and $\left|E\left(\mathrm{DF}_{15}\right)\right|=44$. In accordance with Theorem 13 we have $k=5, m=5, t_{1}=22$, and $t_{2}=20$. Here $t_{1}>t_{2}$ so labeling pattern described in Case 4 will be applicable and $t_{5}=2$. The corresponding prime cordial labeling is shown in Figure 2. Here $e_{f}(0)=22=\mathrm{e}_{f}(1)$.

Illustration 3. For the graph $\mathrm{DF}_{37},\left|V\left(\mathrm{DF}_{37}\right)\right|=39$ and $\left|E\left(\mathrm{DF}_{37}\right)\right|=110$. In accordance with Theorem 13, we have $k=19, m=13, t_{1}=55$, and $t_{2}=57$. Here $t_{2}>t_{1}$ and $n=37$ so labeling pattern described in Sub-Case 2 of Case 5 will be applicable and $t_{6}=2$. And corresponding labeling pattern is as below:

$$
f\left(u_{1}\right)=2, \quad f\left(u_{2}\right)=6 .
$$




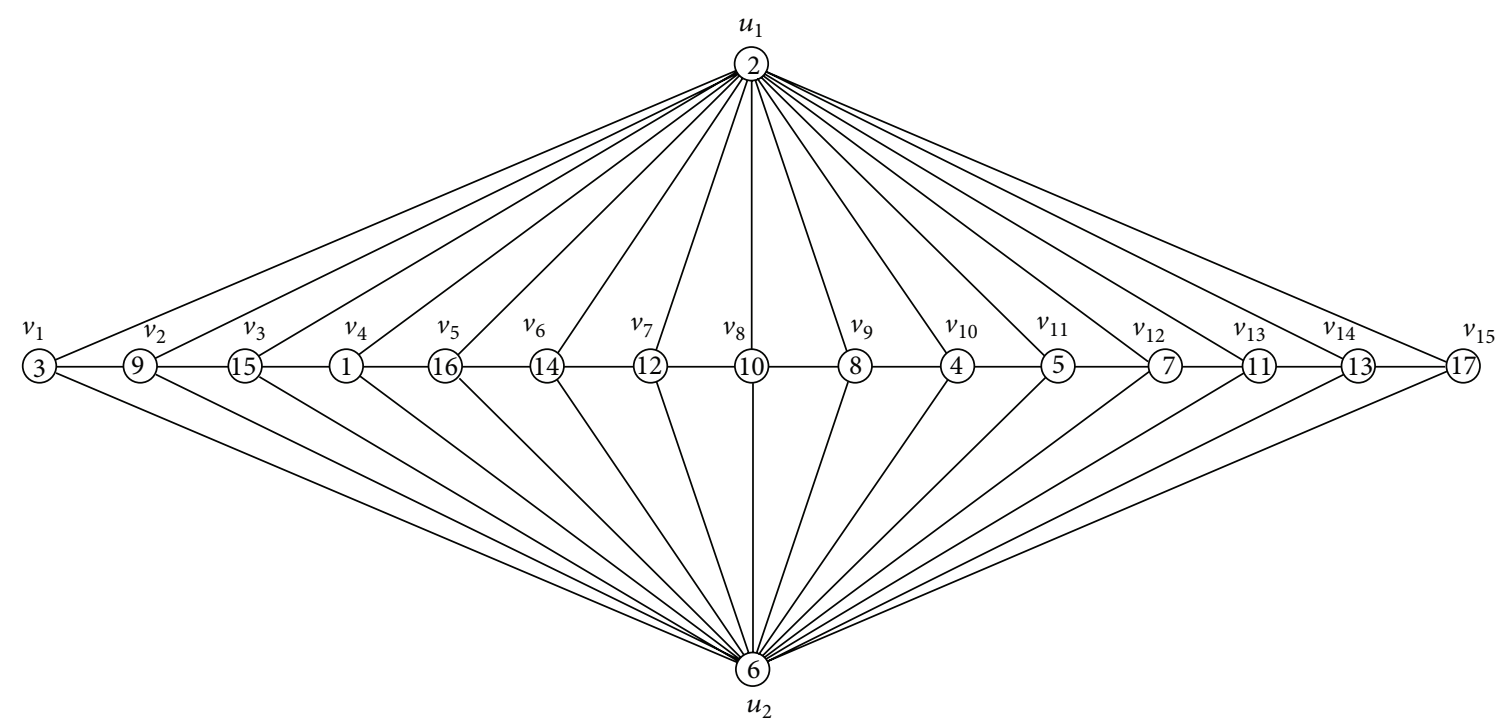

Figure 2

For the vertices $v_{1}, v_{2}, \ldots, v_{37}$ we assign the vertex labels 39, $38,37,36,35,34,32,30,28,26,24,22,20,18,16,14,12,10,8,4$, $3,5,7,9,11,13,15,17,19,21,23,25,27,29,31$, and 33 , respectively, where $e_{f}(0)=55=e_{f}(1)$. Therefore $\mathrm{DF}_{37}$ is a prime cordial graph.

Theorem 14. The graph obtained by duplication of an arbitrary rim edge by an edge in $W_{n}$ is a prime cordial graph, where $n \geq 6$.

Proof. Let $v_{0}$ be the apex vertex of $W_{n}$ and let $v_{1}, v_{2}, \ldots, v_{n}$ be the rim vertices. Without loss of generality we duplicate the rim edge $e=v_{1} v_{2}$ by an edge $e^{\prime}=u_{1} u_{2}$ and call the resultant graph as $G$. Then $|V(G)|=n+3$ and $|E(G)|=2 n+5$. To define $f: V(G) \rightarrow\{1,2,3, \ldots, n+3\}$, we consider the following four cases.

Case $1(n=3,4,5)$. For $n=3$, to satisfy the edge condition for prime cordial labeling, it is essential to label five edges with label 0 and six edges with label 1 out of eleven edges. But all the possible assignments of vertex labels will give rise to 0 labels for at most four edges and 1 label for at least seven edges. That is, $\left|e_{f}(0)-e_{f}(1)\right|=3>1$. Hence, for $n=3, G$ is not a prime cordial graph.

For $n=4$, to satisfy the edge condition for prime cordial labeling, it is essential to label six edges with label 0 and seven edges with label 1 out of thirteen edges. But all the possible assignments of vertex labels will give rise to 0 labels for at most four edges and 1 label for at least nine edges. That is, $\left|e_{f}(0)-e_{f}(1)\right|=5>1$. Hence, for $n=4, G$ is not a prime cordial graph.

For $n=5$, to satisfy the edge condition for prime cordial labeling it is essential to label seven edges with label 0 and eight edges with label 1 out of fifteen edges. But all the possible assignments of vertex labels will give rise to 0 labels for at most six edges and 1 label for at least nine edges. That is, $\left|e_{f}(0)-e_{f}(1)\right|=3>1$. Hence, for $n=5, G$ is not a prime cordial graph.
Case 2 ( $n=6$ to 10). For $n=6, f\left(u_{1}\right)=4, f\left(u_{2}\right)=8$ and $f\left(v_{0}\right)=6, f\left(v_{1}\right)=3, f\left(v_{2}\right)=9, f\left(v_{3}\right)=7, f\left(v_{4}\right)=5$, $f\left(v_{5}\right)=1$, and $f\left(v_{6}\right)=2$. Then $e_{f}(0)=8, e_{f}(1)=9$.

For $n=7, f\left(u_{1}\right)=4, f\left(u_{2}\right)=8$ and $f\left(v_{0}\right)=6, f\left(v_{1}\right)=3$, $f\left(v_{2}\right)=9, f\left(v_{3}\right)=7, f\left(v_{4}\right)=5, f\left(v_{5}\right)=10, f\left(v_{6}\right)=1$, and $f\left(v_{7}\right)=2$. Then $e_{f}(0)=4, e_{f}(1)=5$.

For $n=8, f\left(u_{1}\right)=11, f\left(u_{2}\right)=10$ and $f\left(v_{0}\right)=6, f\left(v_{1}\right)=$ 3, $f\left(v_{2}\right)=2, f\left(v_{3}\right)=8, f\left(v_{4}\right)=4, f\left(v_{5}\right)=5, f\left(v_{6}\right)=7$, $f\left(v_{7}\right)=1$, and $f\left(v_{8}\right)=9$. Then $e_{f}(0)=10, e_{f}(1)=11$.

For $n=9, f\left(u_{1}\right)=11, f\left(u_{2}\right)=12$ and $f\left(v_{0}\right)=2, f\left(v_{1}\right)=$ $1, f\left(v_{2}\right)=3, f\left(v_{3}\right)=6, f\left(v_{4}\right)=8, f\left(v_{5}\right)=4, f\left(v_{6}\right)=10$, $f\left(v_{7}\right)=5, f\left(v_{8}\right)=7$, and $f\left(v_{9}\right)=9$. Then $e_{f}(0)=11, e_{f}(1)=$ 12.

For $n=10, f\left(u_{1}\right)=13, f\left(u_{2}\right)=12$ and $f\left(v_{0}\right)=2$, $f\left(v_{1}\right)=9, f\left(v_{2}\right)=3, f\left(v_{3}\right)=6, f\left(v_{4}\right)=4, f\left(v_{5}\right)=8$, $f\left(v_{6}\right)=10, f\left(v_{7}\right)=5, f\left(v_{8}\right)=1, f\left(v_{9}\right)=7$, and $f\left(v_{10}\right)=11$. Then $e_{f}(0)=12, e_{f}(1)=13$.

Case 3 ( $n$ is even, $n \geq 12$ ). Consider

$$
\begin{aligned}
& f\left(v_{0}\right)=2 \text {, } \\
& f\left(v_{1}\right)=5, \quad f\left(v_{2}\right)=10, \\
& f\left(v_{3}\right)=4, \quad f\left(v_{4}\right)=8, \\
& f\left(v_{4+i}\right)=12+2(i-1), \quad 1 \leq i \leq(n / 2)-5 \\
& f\left(v_{n / 2}\right)=6, \quad f\left(v_{(n / 2)+1}\right)=3, \\
& f\left(v_{(n / 2)+2}\right)=9 \text {, } \\
& f\left(v_{n-1}\right)=1, \quad f\left(v_{n}\right)=7, \\
& f\left(v_{(n / 2)+2+i}\right)=11+2(i-1), \quad 1 \leq i \leq(n / 2)-4 \\
& f\left(u_{1}\right)=n+3, \quad f\left(u_{2}\right)=n+2 .
\end{aligned}
$$


In view of the above defined labeling pattern for Case 3, we have $e_{f}(0)=n+3$ and $e_{f}(1)=n+2$ for $n \equiv 4(\bmod 7)$ and $e_{f}(0)=n+2$ and $e_{f}(1)=n+3$ for $n \not \equiv 4(\bmod 7)$.

Case 4 ( $n$ is odd, $n \geq 11)$. Consider

$$
\begin{aligned}
f\left(v_{0}\right) & =2, \\
f\left(v_{1}\right) & =10, \quad f\left(v_{2}\right)=4, \\
f\left(v_{3}\right) & =8, \\
f\left(v_{3+i}\right) & =12+2(i-1), \quad 1 \leq i \leq((n-1) / 2)-4 \\
f\left(v_{(n-1) / 2}\right) & =6, \quad f\left(v_{(n+1) / 2}\right)=3, \\
f\left(v_{(n+3) / 2}\right) & =1, \\
f\left(v_{n+1-i}\right) & =5+2(i-1), \quad 1 \leq i \leq(n-3) / 2 \\
f\left(u_{1}\right) & =n+2, \quad f\left(u_{2}\right)=n+3 .
\end{aligned}
$$

In view of the above defined labeling pattern for Case 4 , we have $e_{f}(0)=n+3$ and $e_{f}(1)=n+2$ for $n \equiv 3(\bmod 5)$ and $e_{f}(0)=n+2$ and $e_{f}(1)=n+3$ for $n \not \equiv 4(\bmod 7)$.

In view of Cases 2 to 4 we have $\left|e_{f}(0)-e_{f}(1)\right| \leq 1$.

Hence, $G$ is a prime cordial graph for $n \geq 6$.

Illustration 4. Let $G$ be the graph obtained by duplication of an arbitrary rim edge by an edge in wheel $W_{13}$. Then $|V(G)|=16$ and $|E(G)|=31$. In accordance with Theorem 14, Case 4 will be applicable and the corresponding prime cordial labeling is shown in Figure 3. Here $e_{f}(0)=16$, $e_{f}(1)=15$.

Theorem 15. The graph obtained by duplication of an arbitrary spoke edge by an edge in wheel $W_{n}$ is prime cordial graph, where $n=7$ and $n \geq 9$.

Proof. Let $v_{0}$ be the apex vertex of $W_{n}$ and let $v_{1}, v_{2}, \ldots, v_{n}$ be the rim vertices. Without loss of generality we duplicate the spoke edge $e=v_{0} v_{1}$ by an edge $e^{\prime}=u_{1} u_{2}$ and call the resultant graph $G$. Then $|V(G)|=n+3$ and $|E(G)|=3 n+2$. To define $f: V(G) \rightarrow\{1,2,3, \ldots, n+3\}$, we consider following three cases.

Case 1 ( $n=3$ to 6 and $n=8$ ). For $n=3$, to satisfy the edge condition for prime cordial labeling it is essential to label five edges with label 0 and six edges with label 1 out of eleven edges. But all the possible assignments of vertex labels will give rise to 0 labels for at most four edges and 1 label for at least seven edges. That is, $\left|e_{f}(0)-e_{f}(1)\right|=3>1$. Hence, for $n=3, G$ is not a prime cordial graph.

For $n=4$, to satisfy the edge condition for prime cordial labeling, it is essential to label seven edges with label 0 and seven edges with label 1 out of fourteen edges. But all the possible assignments of vertex labels will give rise to 0 labels for at most four edges and 1 label for at least ten edges. That is, $\left|e_{f}(0)-e_{f}(1)\right|=6>1$. Hence, for $n=4, G$ is not a prime cordial graph.

For $n=5$, to satisfy the edge condition for prime cordial labeling, it is essential to label eight edges with label 0 and

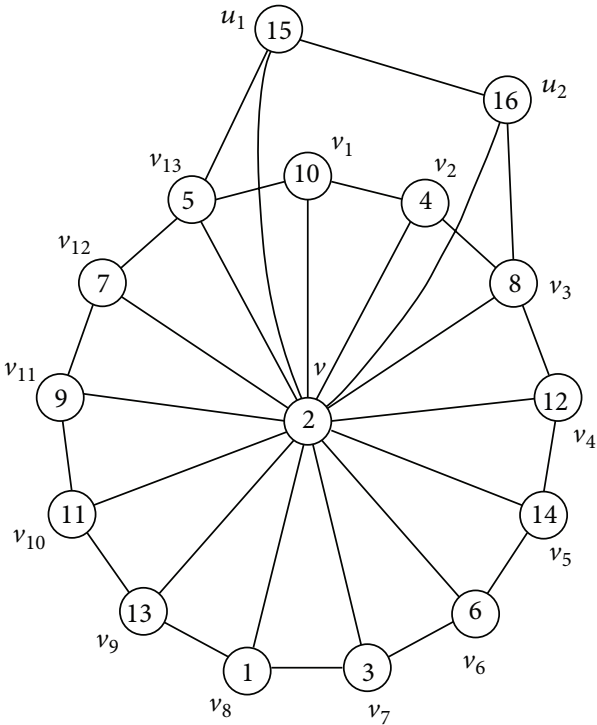

Figure 3

nine edges with label 1 out of seventeen edges. But all the possible assignments of vertex labels will give rise to 0 labels for at most six edges and 1 label for at least eleven edges. That is, $\left|e_{f}(0)-e_{f}(1)\right|=5>1$. Hence, for $n=5, G$ is not a prime cordial graph.

For $n=6$, to satisfy the edge condition for prime cordial labeling, it is essential to label ten edges with label 0 and ten edges with label 1 out of twenty edges. But all the possible assignments of vertex labels will give rise to 0 labels for at most eight edges and 1 label for at least twelve edges. That is, $\left|e_{f}(0)-e_{f}(1)\right|=4>1$. Hence, for $n=6, G$ is not a prime cordial graph.

For $n=8$, to satisfy the edge condition for prime cordial labeling, it is essential to label thirteen edges with label 0 and thirteen edges with label 1 out of twenty-six edges. But all the possible assignments of vertex labels will give rise to 0 labels for at most twelve edges and 1 label for at least fourteen edges. That is, $\left|e_{f}(0)-e_{f}(1)\right|=2>1$. Hence, for $n=8, G$ is not a prime cordial graph.

Case 2 ( $n=7, n=9$ to 12 , and $n=14,16,18,20,22)$. For $n=7, f\left(u_{1}\right)=2, f\left(u_{2}\right)=1$ and $f\left(v_{0}\right)=6, f\left(v_{1}\right)=9$, $f\left(v_{2}\right)=3, f\left(v_{3}\right)=4, f\left(v_{4}\right)=8, f\left(v_{5}\right)=10, f\left(v_{6}\right)=5$, and $f\left(v_{7}\right)=7$. Then $e_{f}(0)=12, e_{f}(1)=11$.

For $n=9, f\left(u_{1}\right)=2, f\left(u_{2}\right)=1$ and $f\left(v_{0}\right)=6, f\left(v_{1}\right)=3$, $f\left(v_{2}\right)=12, f\left(v_{3}\right)=4, f\left(v_{4}\right)=8, f\left(v_{5}\right)=10, f\left(v_{6}\right)=5$, $f\left(v_{7}\right)=7, f\left(v_{8}\right)=11$, and $f\left(v_{9}\right)=9$. Then $e_{f}(0)=15$, $e_{f}(1)=14$.

For $n=10, f\left(u_{1}\right)=2, f\left(u_{2}\right)=1$ and $f\left(v_{0}\right)=6, f\left(v_{1}\right)=$ $3, f\left(v_{2}\right)=12, f\left(v_{3}\right)=4, f\left(v_{4}\right)=8, f\left(v_{5}\right)=10, f\left(v_{6}\right)=5$, $f\left(v_{7}\right)=7, f\left(v_{8}\right)=11, f\left(v_{9}\right)=13$, and $f\left(v_{10}\right)=9$. Then $e_{f}(0)=16, e_{f}(1)=16$.

For $n=11, f\left(u_{1}\right)=2, f\left(u_{2}\right)=1$ and $f\left(v_{0}\right)=6, f\left(v_{1}\right)=$ $5, f\left(v_{2}\right)=4, f\left(v_{3}\right)=8, f\left(v_{4}\right)=10, f\left(v_{5}\right)=14, f\left(v_{6}\right)=12$, $f\left(v_{7}\right)=9, f\left(v_{8}\right)=3, f\left(v_{9}\right)=13, f\left(v_{10}\right)=11$, and $f\left(v_{11}\right)=7$. Then $e_{f}(0)=18, e_{f}(1)=17$. 
For $n=12, f\left(u_{1}\right)=2, f\left(u_{2}\right)=1$ and $f\left(v_{0}\right)=6, f\left(v_{1}\right)=$ $5, f\left(v_{2}\right)=4, f\left(v_{3}\right)=8, f\left(v_{4}\right)=10, f\left(v_{5}\right)=14, f\left(v_{6}\right)=12$, $f\left(v_{7}\right)=9, f\left(v_{8}\right)=3, f\left(v_{9}\right)=13, f\left(v_{10}\right)=15, f\left(v_{11}\right)=11$, and $f\left(v_{12}\right)=7$. Then $e_{f}(0)=19, e_{f}(1)=19$.

For $n=14, f\left(u_{1}\right)=2, f\left(u_{2}\right)=1$ and $f\left(v_{0}\right)=6, f\left(v_{1}\right)=$ $5, f\left(v_{2}\right)=4, f\left(v_{3}\right)=8, f\left(v_{4}\right)=10, f\left(v_{5}\right)=12, f\left(v_{6}\right)=14$, $f\left(v_{7}\right)=16, f\left(v_{8}\right)=3, f\left(v_{9}\right)=9, f\left(v_{10}\right)=15, f\left(v_{11}\right)=17$, $f\left(v_{12}\right)=13, f\left(v_{13}\right)=11$, and $f\left(v_{14}\right)=7$. Then $e_{f}(0)=22$, $e_{f}(1)=22$.

For $n=16, f\left(u_{1}\right)=2, f\left(u_{2}\right)=1$ and $f\left(v_{0}\right)=6, f\left(v_{1}\right)=$ $19, f\left(v_{2}\right)=4, f\left(v_{3}\right)=8, f\left(v_{4}\right)=10, f\left(v_{5}\right)=12, f\left(v_{6}\right)=14$, $f\left(v_{7}\right)=16, f\left(v_{8}\right)=18, f\left(v_{9}\right)=3, f\left(v_{10}\right)=9, f\left(v_{11}\right)=$ $5, f\left(v_{12}\right)=7, f\left(v_{13}\right)=11, f\left(v_{14}\right)=13, f\left(v_{15}\right)=15$, and $f\left(v_{16}\right)=17$. Then $e_{f}(0)=25, e_{f}(1)=25$.

For $n=18, f\left(u_{1}\right)=2, f\left(u_{2}\right)=1$ and $f\left(v_{0}\right)=6, f\left(v_{1}\right)=$ 21, $f\left(v_{2}\right)=4, f\left(v_{3}\right)=8, f\left(v_{4}\right)=10, f\left(v_{5}\right)=12, f\left(v_{6}\right)=14$, $f\left(v_{7}\right)=16, f\left(v_{8}\right)=18, f\left(v_{9}\right)=20, f\left(v_{10}\right)=3, f\left(v_{11}\right)=9$, $f\left(v_{12}\right)=5, f\left(v_{13}\right)=7, f\left(v_{14}\right)=11, f\left(v_{15}\right)=13, f\left(v_{16}\right)=15$, $f\left(v_{17}\right)=17$, and $f\left(v_{18}\right)=19$. Then $e_{f}(0)=28, e_{f}(1)=28$.

For $n=20, f\left(u_{1}\right)=2, f\left(u_{2}\right)=1$ and $f\left(v_{0}\right)=6, f\left(v_{1}\right)=$ 23, $f\left(v_{2}\right)=4, f\left(v_{3}\right)=8, f\left(v_{4}\right)=10, f\left(v_{5}\right)=12, f\left(v_{6}\right)=14$, $f\left(v_{7}\right)=16, f\left(v_{8}\right)=18, f\left(v_{9}\right)=20, f\left(v_{10}\right)=22, f\left(v_{11}\right)=3$, $f\left(v_{12}\right)=9, f\left(v_{13}\right)=5, f\left(v_{14}\right)=7, f\left(v_{15}\right)=11, f\left(v_{16}\right)=13$, $f\left(v_{17}\right)=15, f\left(v_{18}\right)=17, f\left(v_{19}\right)=19$, and $f\left(v_{20}\right)=21$. Then $e_{f}(0)=31, e_{f}(1)=31$.

For $n=22, f\left(u_{1}\right)=2, f\left(u_{2}\right)=1$ and $f\left(v_{0}\right)=6, f\left(v_{1}\right)=$ 25, $f\left(v_{2}\right)=4, f\left(v_{3}\right)=8, f\left(v_{4}\right)=10, f\left(v_{5}\right)=12, f\left(v_{6}\right)=14$, $f\left(v_{7}\right)=16, f\left(v_{8}\right)=18, f\left(v_{9}\right)=20, f\left(v_{10}\right)=22, f\left(v_{11}\right)=24$, $f\left(v_{12}\right)=3, f\left(v_{13}\right)=5, f\left(v_{14}\right)=7, f\left(v_{15}\right)=9, f\left(v_{16}\right)=11$, $f\left(v_{17}\right)=13, f\left(v_{18}\right)=15, f\left(v_{19}\right)=17, f\left(v_{20}\right)=19, f\left(v_{21}\right)=$ 21 , and $f\left(v_{22}\right)=23$. Then $e_{f}(0)=34, e_{f}(1)=34$.

For the next case let $t_{1}=\lfloor(n+3) / 2\rfloor, m=\lfloor(n+3) / 3\rfloor$, $t_{2}=\lceil m / 2\rceil, k_{1}=\lfloor(3 n+2) / 2\rfloor, k_{2}=2 t_{1}+t_{2}-4$,

$$
t=k_{1}-k_{2}, \quad t_{3}= \begin{cases}t-2 ; & n=13,15,17 \\ t-1 ; & n=19,21, \quad n \geq 23 .\end{cases}
$$

Case $3\left(t_{1}-t \geq 3(n=13,15,17,19,21\right.$ and $\left.n \geq 23)\right)$. Consider

$$
\begin{aligned}
f\left(u_{1}\right) & =2, \quad f\left(u_{2}\right)=5, \\
f\left(v_{0}\right) & =6, \\
f\left(v_{1}\right) & =3, \quad f\left(v_{2}\right)=4, \\
f\left(v_{n}\right) & =1, \\
f\left(v_{2+i}\right) & =8+2(i-1), \quad 1 \leq i \leq t \\
f\left(v_{t+3}\right) & = \begin{cases}9, & \text { if } t \equiv 4(\bmod 7) \\
7, & \text { otherwise }\end{cases} \\
f\left(v_{t+4}\right) & = \begin{cases}7, & \text { if } t \equiv 4(\bmod 7) \\
9, & \text { otherwise }\end{cases} \\
f\left(v_{t+4+i}\right) & =9+2 i, \quad 1 \leq i \leq t_{3} .
\end{aligned}
$$

For $2 t+3<n-1$,

$$
f\left(v_{2 t+3+i}\right)=f\left(v_{2 t+3}\right)+i, \quad 1 \leq i \leq(n-1)-(2 t+3) .
$$

In view of the above defined labeling pattern for Case 3, we have $e_{f}(0)=\lfloor(3 n+2) / 2\rfloor$ and $e_{f}(1)=\lceil(3 n+2) / 2\rceil$.

Thus for Cases 2 and 3 we have $\left|e_{f}(0)-e_{f}(1)\right| \leq 1$.

Hence, $G$ is a prime cordial graph for $n=7$ and $n \geq 9$.

Illustration 5. Let $G_{1}$ be the graph obtained by duplication of arbitrary spoke edge by an edge of wheel $W_{23}$. Then $|V(G)|=$ 26 and $|E(G)|=71$. In accordance with Theorem 15 we have $t_{1}=13, m=8, t_{2}=4, k_{1}=35, k_{2}=26$, and $t=9$. Here $t_{1}-t=4>3$ so labeling pattern described in Case 3 will be applicable. The corresponding prime cordial labeling is shown in Figure 4. It is easy to visualise that $e_{f}(0)=35$, $e_{f}(1)=36$.

\section{Theorem 16. $D S\left(P_{n}\right)$ is a prime cordial graph.}

Proof. Consider $P_{n}$ with $V\left(P_{n}\right)=\left\{v_{i}: 1 \leq i \leq n\right\}$. Here $V\left(P_{n}\right)=V_{1} \cup V_{2}$, where $V_{1}=\left\{v_{i}: 2 \leq i \leq n-1\right\}$ and $V_{2}=\left\{v_{1}, v_{n}\right\}$. Now in order to obtain $\operatorname{DS}\left(P_{n}\right)$ from $G$, we add $w_{1}, w_{2}$ corresponding to $V_{1}, V_{2}$. Then $\left|V\left(\mathrm{DS}\left(P_{n}\right)\right)\right|=n+2$ and $E\left(\operatorname{DS}\left(P_{n}\right)\right)=\left\{v_{1} w_{2}, v_{2} w_{2}\right\} \cup\left\{w_{1} v_{i}: 2 \leq i \leq n-1\right\} \cup$ $E\left(P_{n}\right)$ so $\mid E\left(\operatorname{DS}\left(P_{n}\right) \mid=2 n-1\right.$. We define vertex labeling $f: V\left(\operatorname{DS}\left(P_{n}\right)\right) \rightarrow\{1,2, \ldots, n+2\}$ as follows.

Let $p_{1}$ be the highest prime number $<n+2$ and $k=\lfloor(n+$ 2)/2」. Consider

$$
\begin{array}{ll}
f\left(w_{1}\right)=2, & f\left(w_{2}\right)=3, \\
f\left(v_{1}\right)=1, & \\
f\left(v_{n}\right)=9, & f\left(v_{n-1}\right)=p_{1} .
\end{array}
$$

For $0 \leq i<k-1$,

$$
f\left(v_{2+i}\right)= \begin{cases}(n+2)-2 i ; & n \text { is even } \\ (n+1)-2 i ; & n \text { is odd }\end{cases}
$$

And for vertices $v_{k+2}, v_{k+3}, \ldots, v_{n-2}$ we assign distinct odd numbers $(<n+2)$ in ascending order starting from 5 .

In view of the above defined labeling pattern, if $n$ is even number, then $e_{f}(0)=n, e_{f}(1)=n-1$; otherwise $e_{f}(0)=n-1$, $e_{f}(1)=n$.

Thus, $\left|e_{f}(0)-e_{f}(1)\right| \leq 1$.

Hence, $\operatorname{DS}\left(P_{n}\right)$ is a prime cordial graph.

Illustration 6. Prime cordial labeling of the graph $\operatorname{DS}\left(P_{7}\right)$ is shown in Figure 5.

Theorem 17. $D S\left(B_{n, n}\right)$ is a prime cordial graph.

Proof. Consider $B_{n, n}$ with $V\left(B_{n, n}\right)=\left\{u, v, u_{i}, v_{i}: 1 \leq i \leq n\right\}$, where $u_{i}, v_{i}$ are pendant vertices. Here $V\left(B_{n, n}\right)=V_{1} \cup V_{2}$, where $V_{1}=\left\{u_{i}, v_{i}: 1 \leq i \leq n\right\}$ and $V_{2}=\{u, v\}$. Now in order to obtain $\operatorname{DS}\left(B_{n, n}\right)$ from $G$, we add $w_{1}, w_{2}$ corresponding to $V_{1}, V_{2}$. Then $\mid V\left(\operatorname{DS}\left(B_{n, n}\right) \mid=2 n+4\right.$ and $E\left(\operatorname{DS}\left(B_{n, n}\right)\right)=$ $\left\{u v, u w_{2}, v w_{2}\right\} \cup\left\{u u_{i}, v v_{i}, w_{1} u_{i}, w_{1} v_{i}: 1 \leq i \leq n\right\}$ 


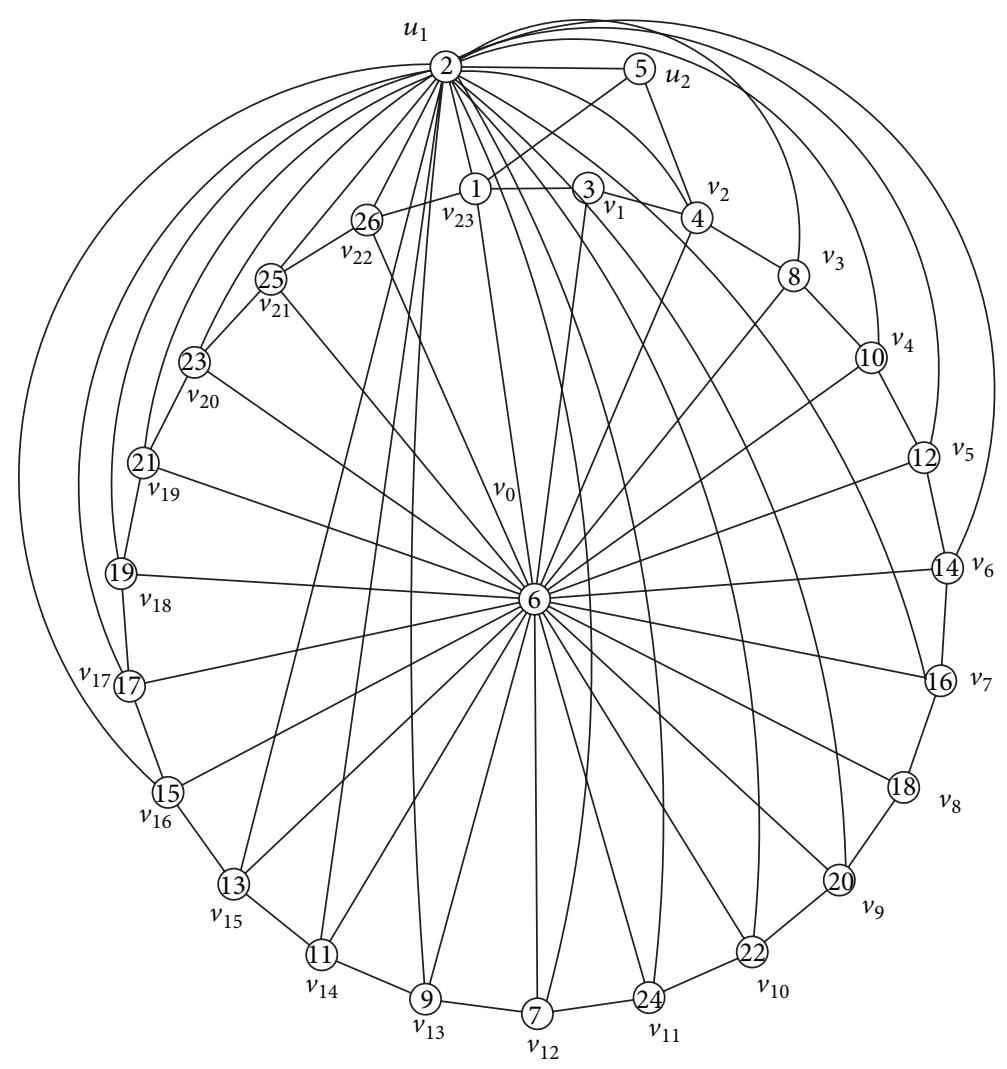

FIGURE 4

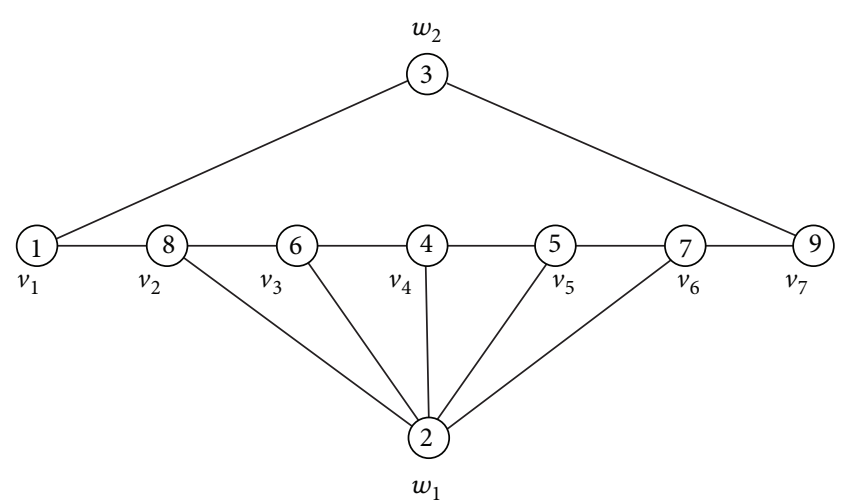

FIgURe 5

so $\mid E\left(\operatorname{DS}\left(B_{n, n}\right) \mid=4 n+3\right.$. We define vertex labeling $f$ : $V\left(D S\left(B_{n, n}\right)\right) \rightarrow\{1,2, \ldots, 2 n+4\}$ as follows:

$$
\begin{aligned}
f(u) & =6, \quad f(v)=1, \\
f\left(w_{1}\right) & =2, \quad f\left(w_{2}\right)=3, \\
f\left(u_{1}\right) & =4, \\
f\left(u_{i+1}\right) & =8+2(i-1), \quad 1 \leq i \leq n-1 \\
f\left(v_{i}\right) & =5+2(i-1), \quad 1 \leq i \leq n .
\end{aligned}
$$

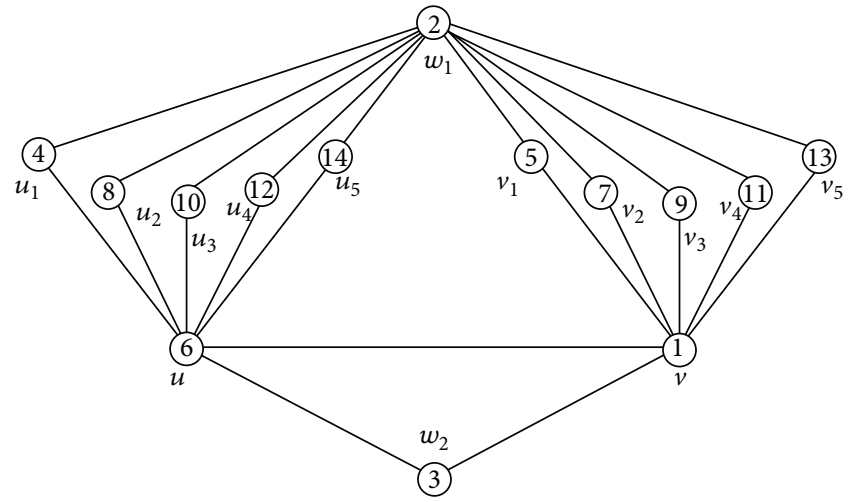

FIGURE 6

In view of the above defined labeling pattern we have $e_{f}(0)=$ $2 n+1, e_{f}(1)=2 n+2$.

Thus, $\left|e_{f}(0)-e_{f}(1)\right| \leq 1$.

Hence, $\mathrm{DS}\left(B_{n, n}\right)$ is a prime cordial graph.

Illustration 7. Prime cordial labeling of the graph $\operatorname{DS}\left(B_{5,5}\right)$ is shown in Figure 6.

\section{Conclusion}

A new approach for constructing larger prime cordial graph from the existing prime cordial graph is investigated. 


\section{Conflict of Interests}

The authors declare that there is no conflict of interests regarding the publication of this paper.

\section{Acknowledgment}

The authors' thanks are due to the anonymous referees for careful reading and constructive suggestions for the improvement in the first draft of this paper.

\section{References}

[1] R. Balakrishnan and K. Ranganathan, A Textbook of Graph Theory, Springer, New York, NY, USA, 2nd edition, 2012.

[2] D. M. Burton, Elementary Number Theory, Brown Publishers, 2nd edition, 1999.

[3] V. Yegnanarayanan and P. Vaidhyanathan, "Some interesting applications of graph labellings," Journal of Mathematical and Computational Science, vol. 2, no. 5, pp. 1522-1531, 2012.

[4] J. A. Gallian, "A dynamic survey of graph labeling," The Electronic Journal of Combinatorics, vol. 16, article DS6, 2013.

[5] I. Cahit, "Cordial graphs: a weaker version of graceful and harmonious graphs," Ars Combinatoria, vol. 23, pp. 201-207, 1987.

[6] A. Tout, A. N. Dabboucy, and K. Howalla, "Prime labeling of graphs," National Academy Science Letters, vol. 11, pp. 365-368, 1982.

[7] S. K. Vaidya and U. M. Prajapati, "Some new results on prime graphs," Open Journal of Discrete Mathematics, vol. 2, no. 3, pp. 99-104, 2012.

[8] S. K. Vaidya and U. M. Prajapati, "Some switching invariant prime graphs," Open Journal of Discrete Mathematics, vol. 2, no. 1, pp. 17-20, 2012.

[9] S. K. Vaidya and U. M. Prajapati, "Prime labeling in the context of duplication of graph elements," International Journal of Mathematics and Soft Computing, vol. 3, no. 1, pp. 13-20, 2013.

[10] M. Sundaram, R. Ponraj, and S. Somasundram, "Prime cordial labelling of graphs," Journal of the Indian Academy of Mathematics, vol. 27, no. 2, pp. 373-390, 2005.

[11] S. K. Vaidya and P. L. Vihol, "Prime cordial labeling for some cycle related graphs," International Journal of Open Problems in Computer Science and Mathematics, vol. 3, no. 5, pp. 223-232, 2010.

[12] S. K. Vaidya and P. L. Vihol, "Prime cordial labeling for some graphs," Modern Applied Science, vol. 4, no. 8, pp. 119-126, 2010.

[13] S. K. Vaidya and N. H. Shah, "Some new families of prime cordial graphs," Journal of Mathematics Research, vol. 3, no. 4, pp. 21-30, 2011.

[14] S. K. Vaidya and N. H. Shah, "Prime cordial labeling of some graphs," Open Journal of Discrete Mathematics, vol. 2, no. 1, pp. 11-16, 2012.

[15] S. K. Vaidya and N. H. Shah, "Prime cordial labeling of some wheel related graphs," Malaya Journal of Matematik, vol. 4, no. 1, pp. 148-156, 2013.

[16] S. Babitha and J. Baskar Babujee, "Prime cordial labeling and duality," Advances and Applications in Discrete Mathematics, vol. 11, no. 1, pp. 79-102, 2013.

[17] S. Babitha and J. Baskar Babujee, "Prime cordial labeling on graphs," International Journal of Mathematical Sciences, vol. 7, no. 1, pp. 43-48, 2013.
[18] P. Selvaraju, P. Balaganesan, J. Renuka, and V. Balaj, "Degree splitting graph on graceful, felicitous and elegant labeling," International Journal of Mathematical Combinatorics, vol. 2, pp. 96-102, 2012. 


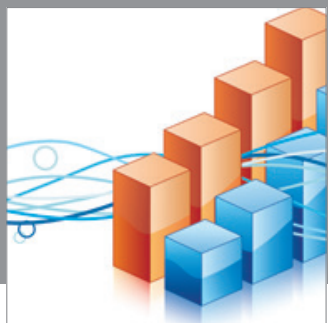

Advances in

Operations Research

mansans

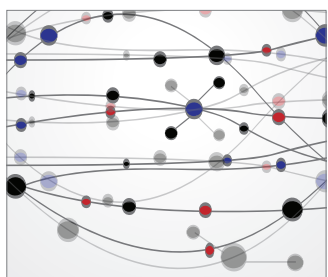

The Scientific World Journal
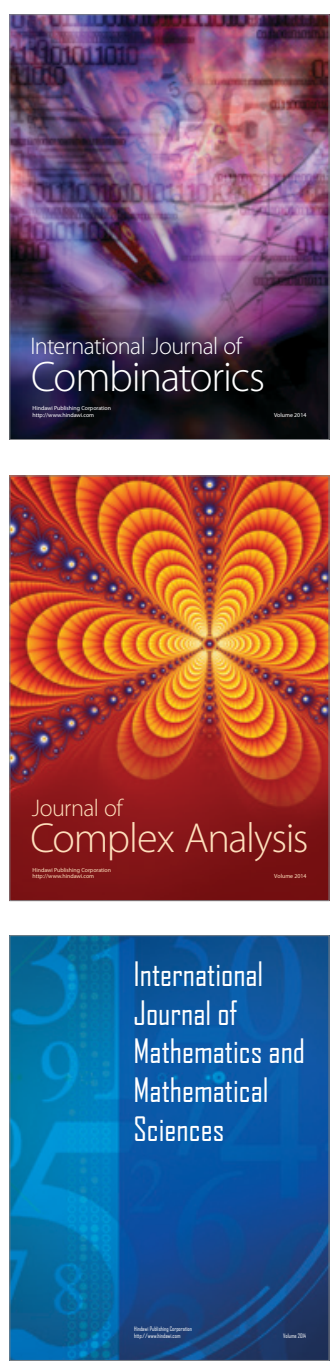
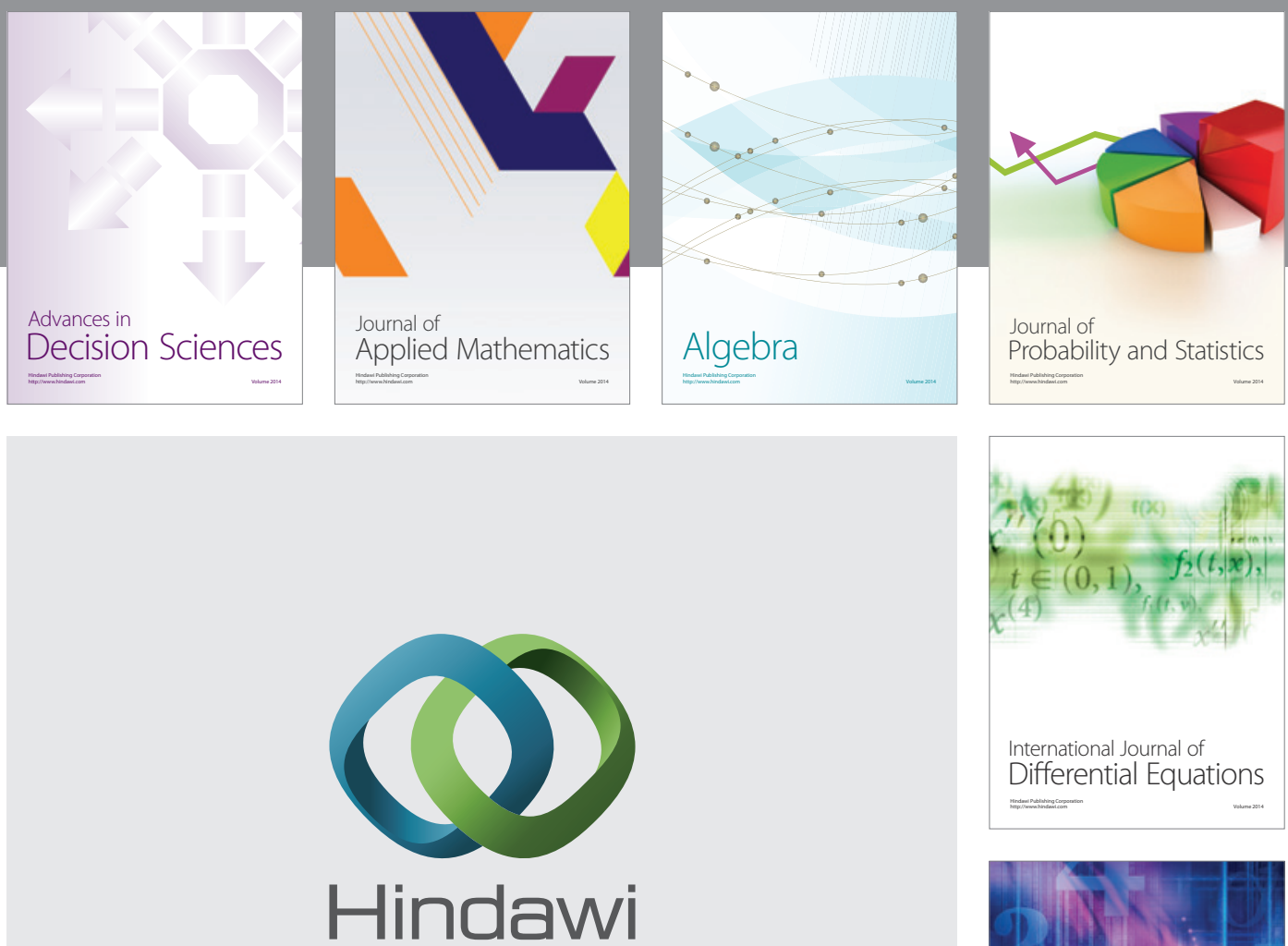

Submit your manuscripts at http://www.hindawi.com
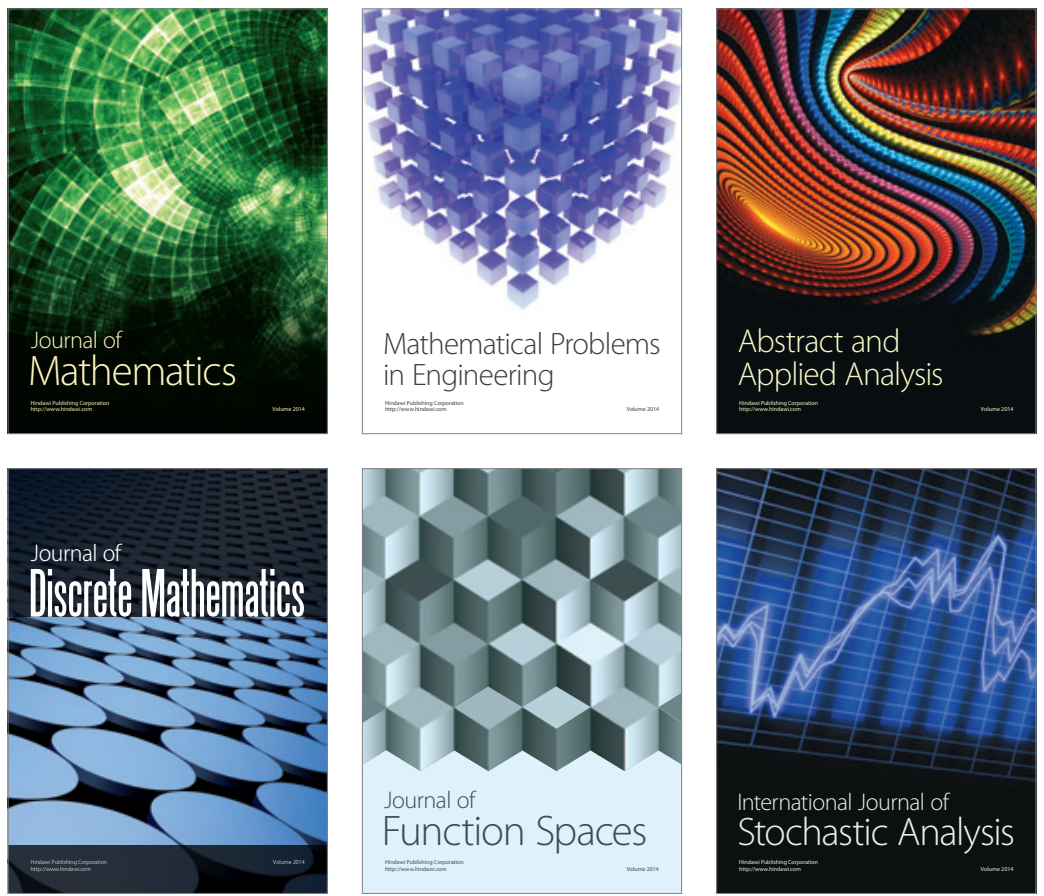

Journal of

Function Spaces

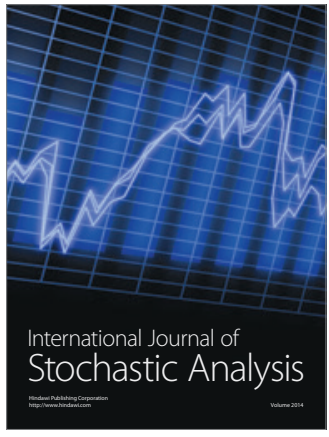

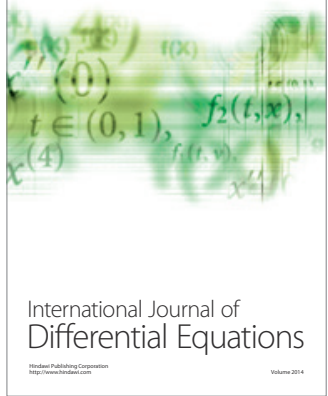
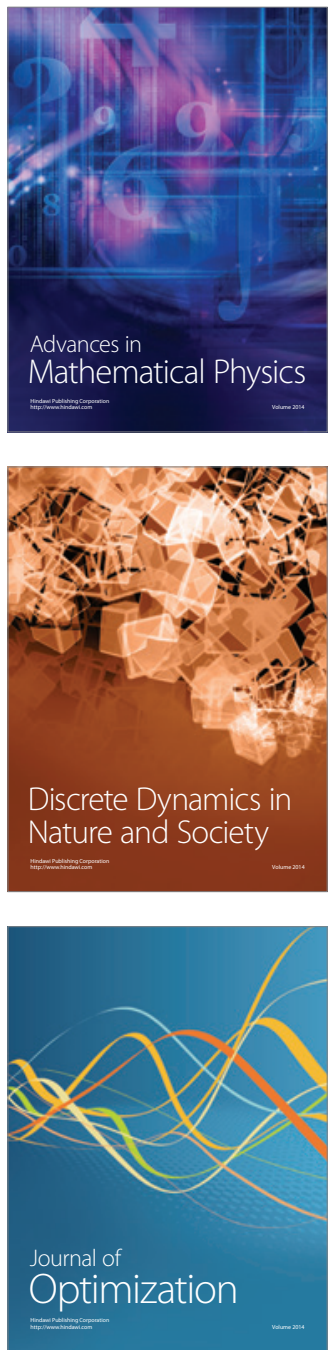